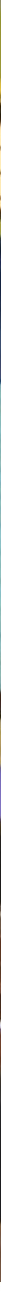

\title{
Virtual Migration through Online Freelancing: Evidence from Bangladesh
}

Case Study

Digital Pathways Paper Series

DOl: https://doi.org/10.35489/BSG-DP-WP_2021/O3

M. Mehrab Bakhtiar, Abu Shonchoy,

Muhammad Meki and Simon Quinn
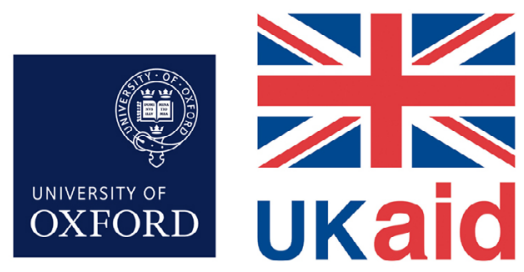

祲

Foreign, Commonwealth \& Development Office

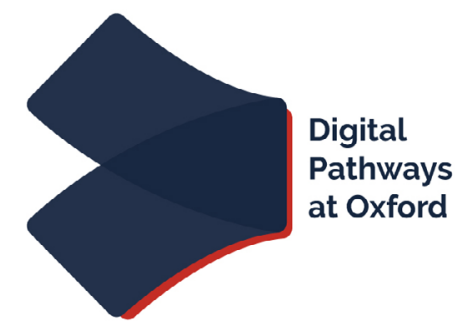




\section{Mehrab Bakhtiar, International}

Food Policy Research Institute:

Abu Shonchoy, Florida International

University; and Muhammad Meki and

Simon Quinn, University of Oxford.

Paper 13

May 2021

Digital Pathways at Oxford is a research programme based at the Blavatnik School of Government, University of Oxford. It produces cutting-edge research across the fields of public policy, law, economics, computer science, and political science to support informed decisionmaking on the governance of digital technologies, with a focus on low- and middle-income countries.

This paper is part of a series of papers on technology policy and regulation, bringing together evidence, ideas and novel research on the strengths and weaknesses of emerging practice in developing nations. The views and positions expressed in this paper are those of the author and do not represent the University of Oxford.

Citation:

Bakhtiar, M. M., Shonchoy, A., Meki, M. and Quinn, S. (2021) Virtual Migration through Online Freelancing: Evidence from Bangladesh. Digital Pathways at Oxford Paper Series; no. 13. Oxford, UnitedKingdom

https://www.bsg.ox.ac.uk/research/ research-programmes/digital-pathways

This paper is licensed under the Creative Commons Attribution 4.0 International License

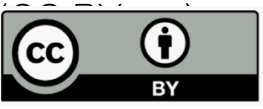

(a)igiPathOxf

Cover image: (c) Jahangir Alam
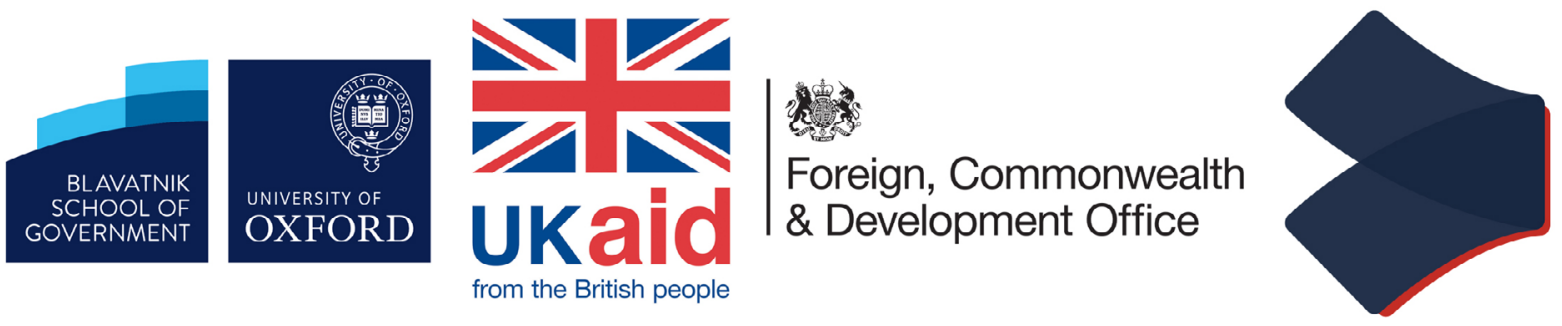


\section{Abstract}

Youth unemployment is a major issue in many developing countries, particularly in locations not well connected with large urban markets. A limited number of available job opportunities in urban centres may reduce the benefit of policies that encourage rural-urban migration.

In this project, we investigated the feasibility of 'virtual migration', by training rural youth in Bangladesh to become online freelancers, enabling them to export their labour services to a global online marketplace. We did this by setting up a 'freelancing incubator', which provided the necessary workspace and infrastructure - specifically, high-speed internet connectivity and computers. Close mentoring was also provided to participants to assist in navigating the competitive online marketplace.

We show the exciting potential of online work for improving the incomes of poor youth in developing countries. We also highlight the constraints to this type of work: financing constraints for the high training cost, access to the necessary work infrastructure, and soft skills requirements to succeed in the market.

We also shed light on some promising possibilities for innovative financial contracts and for 'freelancing incubators' or 'virtual exporting companies' to assist students in their sourcing of work and skills development. 


\section{Table of Contents}

'Virtual migration' to tackle youth unemployment in low-income countries 2

Methodology: Setting up a 'freelancing incubator' 2

Exploring financial constraints to take-up of training programmes 3

Characteristics of our participants 3

Results: Take-up and financing constraints 4

Results: Skills development and income generation 5

Policy implications and future directions 9

$\begin{array}{lll}\text { References } & 11\end{array}$ 


\section{'Virtual migration' to tackle youth unemployment in low-income countries}

Physical migration out of rural areas is not always a viable option for many young people in lowincome countries, often due to limited urban job opportunities and family obligations (Bryan, Chowdhury and Mobarak, 2014; Levy and Sri-Raman, 2018; Shonchoy, Fuji, and Raihan, 2018). The spatial disparity in opportunities can exacerbate the incidence of youth unemployment and poverty, particularly in regions that are not well connected with large urban markets. For example, most of the jobs in the ready-made garment sector - widely considered as the main driver of growth and employment in Bangladesh - are concentrated in Dhaka and in the port city of Chittagong. The poorest regions in Bangladesh, such as the northern district of Rangpur - where we implemented our work - have the lowest participation rate for workers in the garment sector (Shonchoy, Fuji and Raihan, 2018).

In this project, we explored the potential for 'virtual migration': training rural youth to become online freelancers, which enables them to export their labour services to a global online marketplace. Online freelancing platforms provide opportunities for skilled and semi-skilled freelancers from developing countries to earn internationally competitive wages and gain valuable work experience. Such work also provides employment opportunities for those who are often discriminated against - for example, women and individuals with physical disabilities. Working online in marketing and communications, these employees can earn a competitive wage in a market where success is primarily a function of the quality of one's work portfolio. Online work can be very profitable for low-income individuals, but training programmes are often very expensive.

\section{Methodology: Setting up a 'freelancing incubator'}

With the help of local research collaboration partners, Gana Unnayan Kendra (GUK) and MOMODa Foundation, as well as a large ICT company Creative Institute, we began the project in January 2018, implementing it in two waves.

In the first wave, we began by providing a 'theory only' programme: a detailed training course that provided participants with the technical skills necessary to conduct online freelancing work. During the first wave, we observed that - while the students were indeed acquiring some useful technical skills (specifically in graphic design) - their communication and marketing skills were likely to inhibit their ability to succeed in the competitive online marketplace.

Therefore, in the second wave, we decided to include a post-training internship, with trainers providing additional support to the students in navigating the online marketplace, building up online profiles (for example, by taking part in 'contests' to demonstrate their skills) and in securing jobs. Finally, we also went into the field two years later (July 2020) to explore longer-term outcomes for our participants. 


\section{Exploring financial constraints to take-up of training programmes}

Online freelancing training programmes are often very expensive. And, given the volatile incomes streams associated with online work, (particularly in the early stage of career progression), our study included incentivised activities to explore financing constraints and novel financing contracts that would allow participants to share the risks.

We randomly offered participants different payment options for the relatively high cost of the training programme (approximately US\$100):

- Option 1: making the full upfront payment (with some probability of being offered a partial subsidy)

- Option 2: a zero-interest loan to finance the training fee (we also varied the tenor of the loan)

- Option 3: income-sharing contracts, where participants make monthly payments calculated as a share of the income they earnt once they started working on freelancing platforms (rather than a fixed monthly payment, as in the loan). We implemented different income-sharing ratios, ranging from $40 \%$ to $70 \%$. These ratios were randomly allocated across two different groups of students, and we also varied the ratios over time. Payments were implemented based on the income that we directly observed participants earning on the platform.

We implemented an incentivised task to explore demand for different types of financing contract before beginning the actual training programme. In the first wave of the programme, we implemented options 1 and 2, and in the second wave we implemented option 3's income-sharing contracts. We also collected administrative data on student performance throughout the pilot.

\section{Characteristics of our participants}

At the start of the project in 2018, we recruited participants from local colleges in Gaibandha, and also used social media. We received applications from 373 students (across the two waves), out of which 129 passed the minimum computer literacy screening exam from our ICT company collaborator and were permitted to enter the programme.

We conducted a baseline survey with those individuals. Some summary statistics are presented in Table 1. The average age was 22, and 83\% of participants were male. Only 5\% reported themselves to be the "household head", and 18\% were married. Individuals had an average monthly personal income of US\$24 (with the median person not earning any monthly income; this is unsurprising since $65 \%$ reported that they were students). 
Individuals came from households with an average of five adults. The average total monthly household incomewas US\$193 (median US\$120), while the average of total household consumption expenditure was very similar (mean US\$212, median US\$152). This highlighted the tight financial conditions that many households were in, and also that the typical course fee for online freelance training programmes (usually between US\$100 and US\$200) represented a large proportion of total household income, and is probably an unfeasible investment for most households, given the uncertainty around earnings.

Table 1: Summary statistics

\begin{tabular}{l|c|c|ccc}
\hline \hline & & & & \\
& Mean & Standard deviation & 25th percentile & Median & 75th percentile \\
\hline Age & 22 & 6 & 18 & 21 & 24 \\
Personal income & 24 & 54 & 0 & 0 & 25 \\
Household size & 5 & 2 & 4 & 5 & 7 \\
Household income & 193 & 212 & 38 & 120 & 253 \\
Household expenditure & 212 & 180 & 91 & 152 & 241 \\
\hline \hline
\end{tabular}

Note: All numerical amounts are in US\$, based on the exchange rate at baseline of 79 Bangladeshi taka to 1 US\$.

\section{Results: Take-up and financing constraints}

From our incentivised exercises to explore the importance of credit constraints for participating in such freelance training programmes, we found:

- 51\% take-up by individuals who were offered no subsidy or financing contract and had to pay the full upfront cost

- $87 \%$ take-up by those who were offered a subsidy of $50 \%$ of the programme cost

- $76 \%$ take-up for the six-month zero-interest rate loan

- $98 \%$ take-up when the loan duration was extended to 12 months.

It was clear from this exercise that credit and liquidity constraints are a barrier to enrolment in such programmes.

Unfortunately, in practice, there was a low instance of the upfront fee and loan contracts being repaid in full. In hindsight, this was not surprising, given the low baseline income that many had at the time (as seen in Table 1). We also learnt that it took students a significant amount of time to begin earning income on the platform. This meant that, even those students who had intended to pay off loans with freelance earnings, were not able to earn quickly enough to do so. Many students struggled to meet their required payments, even with a subsidy or loan, and some dropped out. Of the 38 students who initially enrolled in the first wave: 21 completed the training programme; 11 students continued to the internship phase; and 9 of these finished the six-month internship.

In the second wave, we offered income-sharing contracts, and observed a high take-up rate of 84\%: 52 students initially enrolled under the income-sharing contract; 25 completed the training programme; 20 continued to the internship phase; and 15 completed the programme. This is 
also consistent with the results we saw in a 'laboratory-style' experiment we had conducted with participants at baseline, where we had tested the demand for debt contracts compared to a series of income-sharing contracts with various sharing ratios; we found strong evidence of 'debt aversion' and a preference for income-sharing contracts (even when participants were offered an income-sharing contract that required them to share a relatively high proportion of their earnings).

Overall, we observed higher repayment rates for the income-sharing contracts than the debt contracts. This was not surprising, since the income was taxed at source (based on earnings on the platform).

\section{Results: Skills development and income generation}

Our high-frequency surveys during the pilot revealed that several of the trainees had developed significant technical skills. This allowed them to start earning relatively large sums of money on the online marketplace by the end of the training and internship programme. Students participated in a combined total of approximately 580 contests, earning $\$ 750$ (as a group) by the endline survey. Reassuringly, the performance improved over time as we refined the structure of the internship and optimised the mentoring and bidding assistance process for students.

\section{Follow-up survey of participants}

In July 2020, we conducted a long-run follow-up survey with our sample, approximately two years after the pilot. We were able to recontact $85 \%$ of the original sample participants. We also included surveys for several individuals who had graduated from similar training programmes (focused on graphic design) who we learnt about during our work. These graduates came from two distinct groups:

(i) a large 'virtual exporter' of freelance-type work to international clients (the Sarah Institute of eGeneration), mostly in the form of graphic design projects. Out of a possible base of 339 people, we interviewed 284 trainees from the Sarah Institute of eGeneration. ${ }^{1}$ The Institute's training was similar to ours, although it had less of an entrepreneurial focus on running one's own enterprise through an online platform. ${ }^{2}$

(ii) from an earlier pilot study conducted by some of the members of the current research team. This pilot did not involve an internship phase, but was conducted in collaboration with the same ICT company. We surveyed 52 individuals, although the response rate was lower in this sample. ${ }^{3}$

We attempted to contact 339 people: 50 phone numbers were non-active; and only a handful of people refused to participate

We are now working with the Sarah Institute of eGeneration on a new project.

3 We attempted to contact approximately 150 people who had previously completed the earlier training programme; around one third had the phone disconnected or turned off, and another one third did not pick up the phone; 30 people explicitly declined to participate. 


\section{Types of freelancing work}

In total, we surveyed 436 individuals. Of those, $28 \%$ had earnt some income in the last six months from what we define as "freelancing-type work", which was composed of three different types of activity:

(i) Freelancing on a website or online platform

(ii) Freelancing-type work directly for a client (that is, not on a website or online platform; $54 \%$ who did that type of work reported that they have maintained a long-term relationship with those clients)

(iii) Freelancing-type work for a company that receives outsourcing orders.

\section{Average income for freelancers}

The average income for those who were doing some type of freelancing work was quite high, at a mean of US\$106 per month (median US\$88), with a right-skewed distribution as illustrated in Figure 1.

Figure 1: Distribution of monthly income

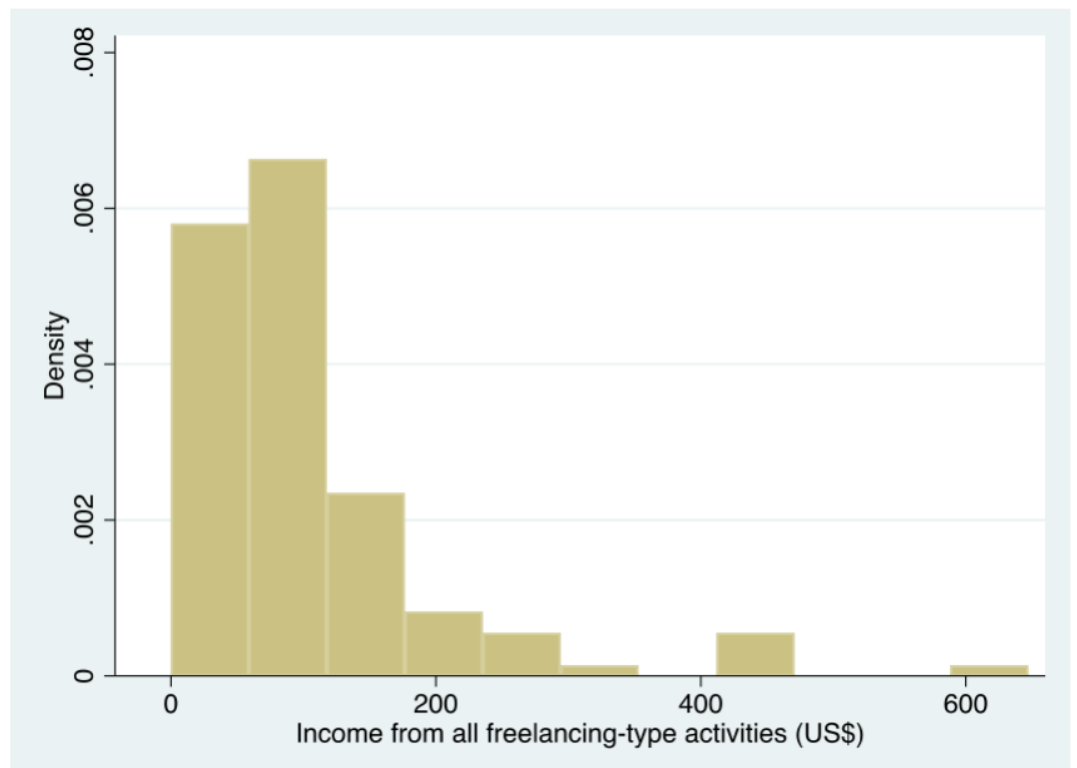

\section{Types of freelance work and online platforms}

Exactly $50 \%$ of those who were earning an income had established an online freelancing profile. Freelancers tended to be active on several platforms, with $67 \%$ having a presence on Freelancer. com $\AA, 37 \%$ on Fiverr and $28 \%$ on Upwork. ${ }^{\mathrm{TM}}$ While it was reassuring to see participants using multiple platforms, it highlighted the difficulty in implementing income-sharing contracts outside of the programme period, given the variety of online platforms that people were earning from. There was also an issue with some people having 'taken their client offline' and had started doing tasks direct for them. 
From our original sample, 34\% had earnt any income from freelancing-type work in the previous six months, while the proportion from the new sample was $26 \%$. Conditional on earning any freelancing-type income, our original sample was significantly more likely to be freelancing on an online platform ( $76 \%$ compared to $21 \%$ for the additional sample). This is unsurprising given the nature of our training (which had an extended hands-on internship built into the design). In contrast, conditional on earning any freelancing-type income, only $5 \%$ of our original group were earning income from work directly for a client (compared to $49 \%$ in the wider sample), and only $22 \%$ were doing freelancing-type work for a company that sources work (compared to $83 \%$ in the wider group, and $87 \%$ in the Sarah Institute of eGeneration sample).

\section{Time worked by freelancers}

Some simple statistical tests revealed that individuals who were earning freelancing income were working significantly more hours than those not engaged in freelancing work (42 hours per week compared to 23 hours per week). Men were much more likely to be earning freelancing income than women (34\% of men had earnt some income from freelancing-type activities in the previous six months, compared to only $8 \%$ of women).

\section{Hours worked and online platform experience}

To further explore earnings for individuals doing freelancing work, we created a variable for 'freelancing experience' based on the length of time they had an active account with an online freelancing platform. Approximately half of those who had earnt any type of freelancing-like income had opened an account with an online platform at some point. This suggested that the remainder had earnt their income through direct contracts with clients or with a company outside of one of the traditional platforms. (There is also a possibility that they were earning platformbased income using someone else's profile, which anecdotal evidence in our context suggests was not common, although we are not able to verify this.)

For those who had opened an online account, the mean number of years of 'platform experience' (active online account) was 3 years (with a median of 2.5 years). We also created a variable for "client experience", based on the time since they first started working directly for clients. The (conditional) mean years of experience was 1.9, with a median of 1.3 years.

A 'scatter plot' and 'line of best fit' reveals a positive relationship between experience - both platform-based and client-based experience - and earnings for individuals involved in freelancing work. This highlights the fact that there are significant gains to be made from this type of work, and earnings do increase over time and with experience. It is important to note that this relationship is for a select group - those who decide to continue working on the platform (who may have a different earning potential than those who did not continue) - and should not be interpreted as the causal effect of having more platform experience. 
Figure 2: Relationship between experience and income
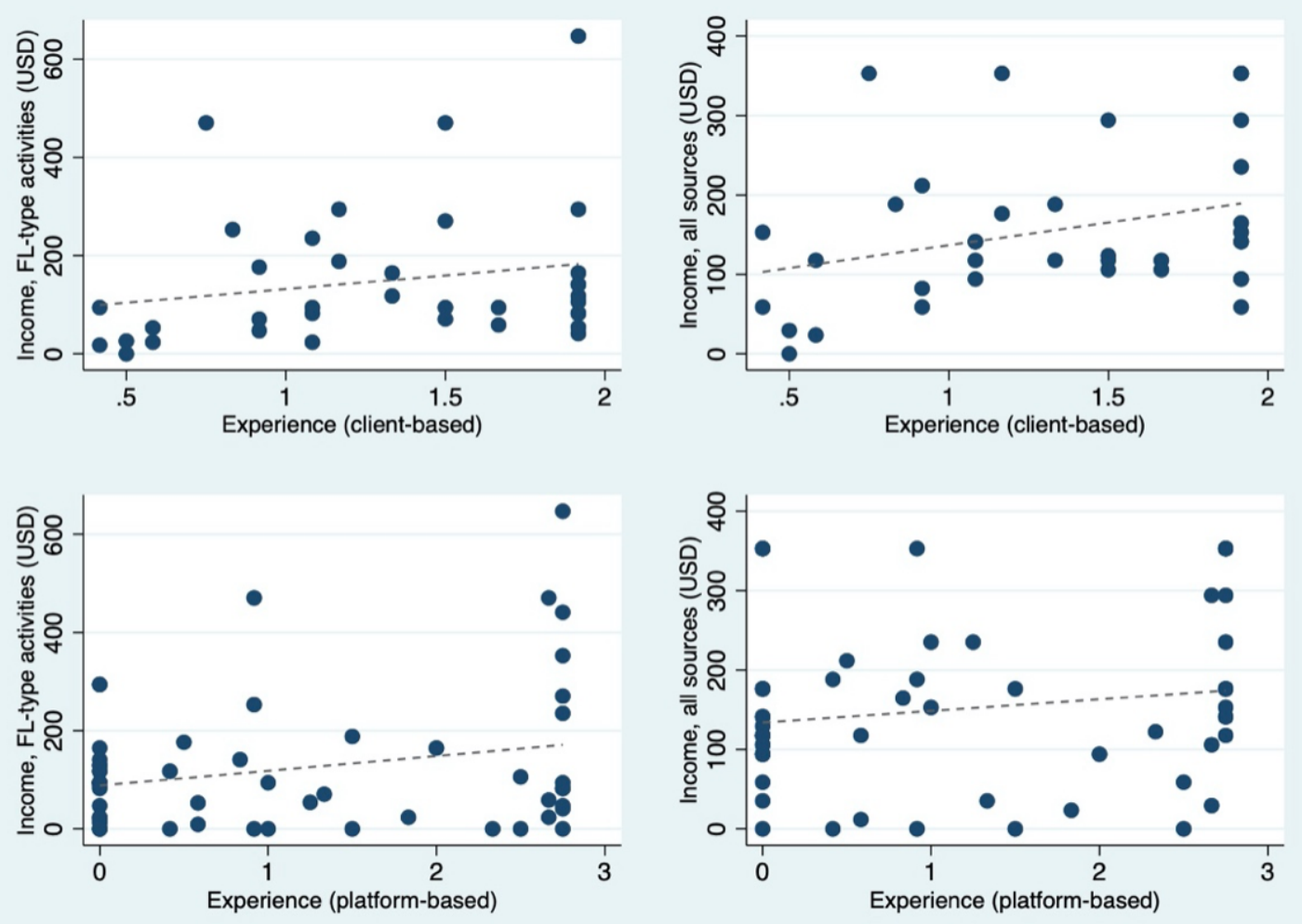

We also analysed more formally the conditional correlations between earnings and experience, while controlling for other potentially relevant characteristics, using regressions. We confirm that the positive correlation observed graphically between experience and earnings is statistically significant, and robust to controlling for other potentially important covariates such as age, gender. marital status and whether the individual is the head of the household. Across various regressions, this indicates that, on average, an extra year of experience is associated with US\$37 to US\$59 of extra monthly income, which is an economically meaningful amount for this population.

Finally, we looked at total hours worked per week as a variable of interest, which has a mean of 28 hours. Here we find that those with more freelancing-type experience work significantly more hours per week than those who do not.

\section{Skills developed by freelancers}

To explore skills development, we asked in our follow-up survey about the main type of skill that individuals who were earning were using: 92\% said graphic design skills. This is unsurprising given the focus on graphic design in the training programmes. Additionally, $6 \%$ had earnt income from search engine optimisation (SEO) and online marketing activities, with 3\% earning from data entry tasks.

We asked those who were earning income from any freelancing-type work what the main challenges were, and the most popular responses were: 
- internet accessibility/stability (51\%)

- English language skills (35\%)

- availability of a computer/workstation (34\%)

- electricity disruptions (20\%).

Other challenges mentioned include: finding the required fee to register for freelancing platforms; difficulty in finding a suitable 'work environment'; the need for more technical training; transportation issues; and time commitments for other work/family tasks. The pie chart in Figure 3 shows the distribution of reported constraints.

Figure 3: Reported work constraints

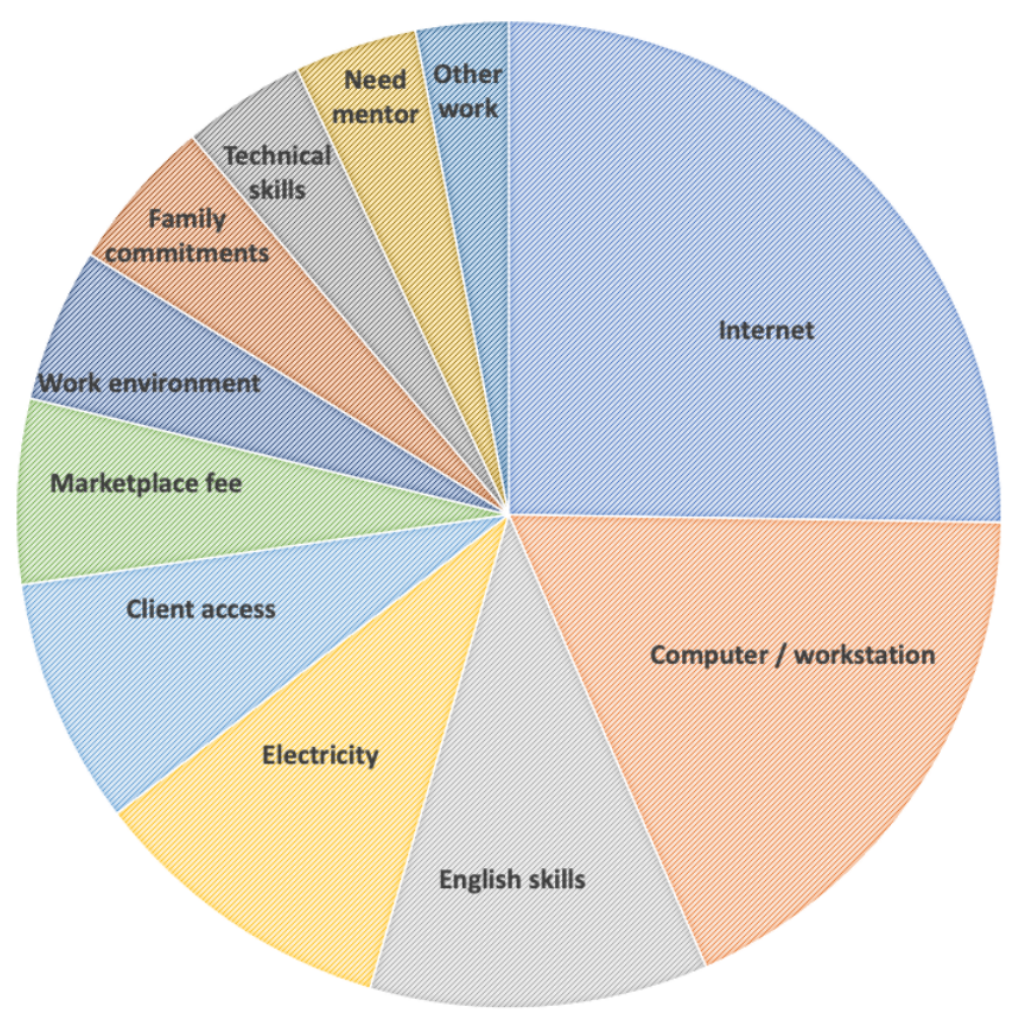

\section{Policy implications and future directions}

There is substantial policy interest in online freelancing from the Bangladesh government. Under the Digital Bangladesh platform, the government plans to train thousands of unemployed people to become freelancers in three key ICT areas:

- graphic design

- web design

- digital marketing. 
The potential stakeholders of this research are the ICT Ministry, Ministry of Planning, Ministry of Labour and Employment, Ministry of Industries, as well as international agencies such as the World Bank, Asian Development Bank, Japan International Cooperation Agency and International Labour Organization.

In general, our findings suggest that online work has exciting potential, but the transition from freelancing student to income-earner is not smooth - it requires financing the significant training programme cost, it takes time to bear fruit (with a large proportion of people not succeeding in earning), and there are a number of constraints.

Our study demonstrated strong technical skill acquisition, but significant challenges from communication skills (particularly in terms of English language and marketing skills), infrastructure challenges (an appropriate work environment, reliable electricity and high-speed internet). We managed to overcome some of these constraints by establishing a 'freelancing incubator', which provided the necessary workspace and infrastructure, as well as the required mentoring to assist in navigating the competitive online marketplace and building a reputation.

Moving forward, we believe that there is potential for many young rural poor to earn income from online work, but more research is needed on how to overcome the significant barriers in terms of financing constraints, soft skill requirements, and more structural challenges such as access to the necessary infrastructure. We found some promise for income-sharing contracts, but making these more financially feasible for the capital provider would require a larger number of students to earn income from such programmes. It was also involve overcoming the significant monitoring challenges to implementing performance-contingent repayments. We are currently conducting more research on this, partnering with the Sarah Institute of eGeneration, who are a large, reputable 'virtual exporter' that sources work globally, and then hires students to complete the work. ${ }^{4}$

This project is funded by an exploratory research grant from Private Enterprise Development in Low-income Countries, a joint research initiative of the Centre for Economic Policy Research and the UK's Foreign, Commonwealth \& Development Office. 


\section{References}

Bryan, G., S.Chowdhury, \& A.Mobarak (2014): Underinvestment in profitable technology: Seasonal migration in Bangladesh. Econometrica, Vol. 82, No. 5 (September, 2014). 1671-1748

Givewell (2018): Update on No Lean Season's top charity status. Blog 19 November 2018. https://blog.givewell.org/2018/11/19/update-on-no-lean-seasons-top-charity-status/

Levy, K. and Sri-Raman, V. (2018): Why (and when) we test at scale: No Lean Season and the quest for impact. Evidence Action Blog, November 2018. https://www.evidenceaction. org/why-test-at-scale-no-lean-season

Shonchoy, A., Fujii, T., \& Raihan, S. (2018). Barriers to Labor Migration for the Rural Poor: Experimental Evidence from a Vocational Training Program in Bangladesh. SSRN Electronic Journal DOI:10.2139/ssrn.3395229. 

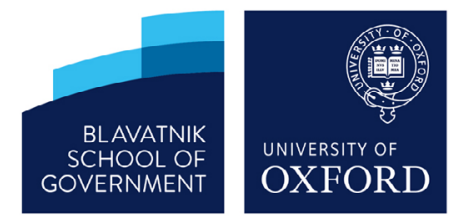

OXFORD
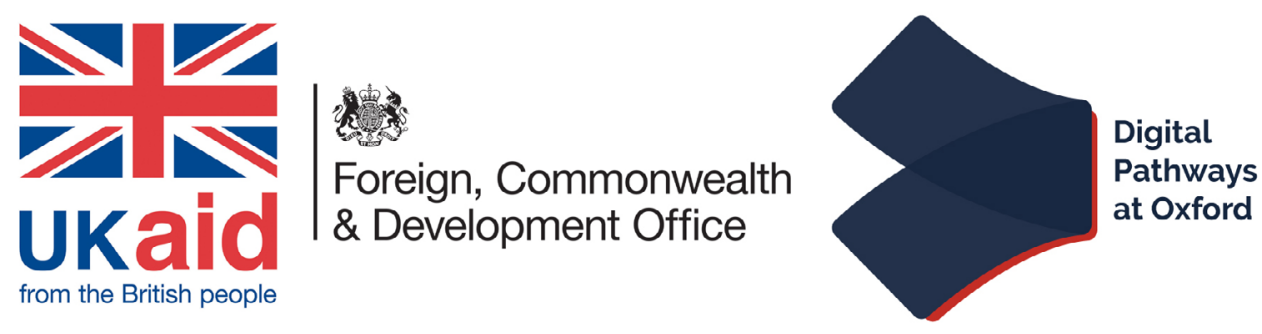